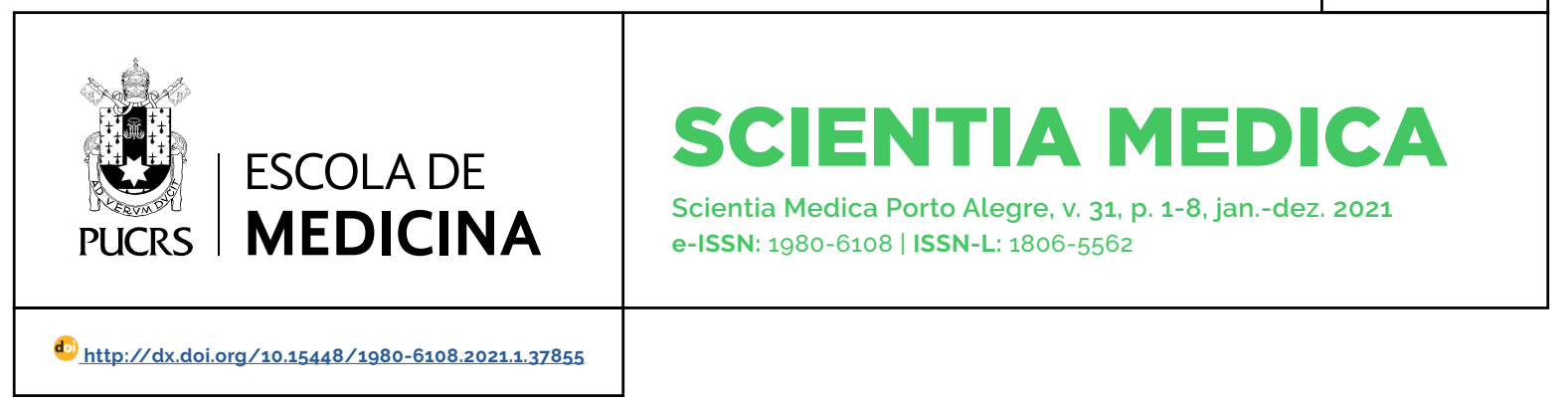

ARTIGO ORIGINAL

\title{
Circunferência do pescoço e fatores de risco cardiovascular em crianças e adolescentes
}

\author{
Neck circumference and cardiovascular risk factors in children and adolescents
}

\section{Ávilla Monalisa Silva de Oliveira $^{1}$ \\ orcid.org/0000-0002-9245-694X avilla.monalisa@hotmail.com}

\section{Skalyt Lee Barbosa e Silva ${ }^{1}$}

orcid.org/0000-0001-7649-6258

skalyt_lee@hotmail.com

\section{Lilian Caroline de Souza e Silva ${ }^{1}$}

orcid.org/0000-0001-6873-026X

Lilian_carolinne@hotmail.com

\section{Ilma Kruze Grande de Arruda ${ }^{2}$}

orcid.org/0000-0002-7142-1967

ilmakruze@hotmail.com

Recebido em: 28 fev. 2020 Aprovado em: 14 fev. 2021. Publicado em: 18 mar. 2021.

\section{(c) (i)}

Artigo está licenciado sob forma de uma licença Creative Commons Atribuição 4.0 Internacional.

\section{Resumo}

Objetivos: Investigar a associação entre a circunferência do pescoço (CP) e fatores de risco cardiovascular em crianças e adolescentes atendidos ambulatorialmente em um hospital universitário na cidade de Recife, PE, Brasil.

Métodos: Foram coletados dados sociodemográficos, antropométricos, bioquímicos, histórico familiar e estilo de vida. A amostra foi por conveniência, composta por crianças e adolescentes acompanhados de seus responsáveis, que aceitassem participar da pesquisa, no periodo de abril a setembro de 2018, no ambulatório de nutrição/pediatria.

Resultados: A amostra foi composta por 144 pacientes com idade média de 11,0 $\pm 3,0$ anos, sendo 73 do sexo masculino (51,4\%). 54 (37,6\%) pacientes apresentaram CP elevada, dentre eles $59(41,1 \%)$ eram do sexo masculino. Foi visto que $102(71,5 \%)$ pacientes apresentaram tempo de tela elevado. Houve associação entre CP e estado nutricional e $13(24,8 \%)$ indivíduos que tinham CP elevada apresentaram também excesso de peso. Foi observado correlação entre circunferência do pescoço com índice de massa corporal e perfil lipídico.

Conclusão: Observou-se que há associação significativa entre CP e algumas medidas antropométricas como IMC e CC, sendo útil para identificação de risco de sobrepeso e de risco cardiovascular. Algumas limitações no estudo podem ser citadas como o limitado número de participantes por ser uma pesquisa transversal, não podendo assim ser estabelecidas medidas de causa e efeito.

Palavras-chave: obesidade, doenças cardiovasculares, estado nutricional.

\section{Abstract}

Aims: To investigate the association between neck circumference (NC) and cardiovascular risk factors in children and adolescents seen in outpatient clinic in a university hospital in the city of Recife-PE.

Methods: Sociodemographic, anthropometric, biochemical and lifestyle data were collected. The sample consisted of convenience, composed of children and adolescents monitored at the nutrition / pediatrics clinic who agreed to participate in the research, from April to September 2018.

Results: The sample consisted of 144 patients with a mean age of $11.0 \pm 3.0$ years, the majority being male (51.4\%). $37.6 \%$ of the patients had elevated NC, most of them male $(41.1 \%)$. A large percentage of patients with high screen time $(71.5 \%)$ were seen. There was a significant association between NC and nutritional status, $24.8 \%$ of those with excess weight presented NC elevation. Positive correlation was found between NC with body mass index and lipid profile.

Conclusion: It was observed that there is a significant association between NC and nutritional status, corroborating with the literature, which suggests that such a measure can be a useful tool to identify overweight or obesity and an important predictor of cardiovascular health problems, since it also correlates with biochemical parameters.

Keywords: obesity, cardiovascular diseases, nutritional status. 
ABREVIATURAS: CC, circunferência da cintura; $C P$, circunferência do pescoço; $\mathrm{CT}$, colesterol total; HDL, lipoproteina de alta densidade (high density lipoprotein); IMC, indice de massa corporal; LDL, lipoproteína de baixa densidade (low density lipoprotein); NC, neck circumference RCE, Relação cintura estatura; VLDL, lipoproteinas de muito baixa densidade (very low density lipoprotein).

\section{Introdução}

O excesso de peso na população brasileira apresentou um crescimento de 23\% entre 2005 e 2015, resultando em um expressivo aumento da incidência e da prevalência desse distúrbio nutricional (1). Portanto, quanto maior for essa prevalência, maior é o interesse no estudo de grupos populacionais mais vulneráveis, como os adolescentes, pela maior probabilidade de manterem o padrão de obesidade na vida adulta (2).

As doenças cardiovasculares têm um longo periodo de latência, porém o surgimento dos fatores de risco é precoce. Nota-se que, durante a adolescência, a presença de dois ou mais fatores de risco é suficiente para a predição de evento cardiovascular nos próximos 10 anos (3) Estudiosos vêm destacando a importância de diagnosticar esses riscos o quanto antes, devido à ascendência desse tipo de doenças em crianças e adolescentes nos últimos anos.

Várias técnicas são utilizadas para mensurare avaliar a distribuição de gordura corporal, como a tomografia computadorizada, a densitometria por dupla emissão de raios- $X$, o índice de massa corporal (IMC), as dobras cutâneas e a circunferência da cintura (CC); entretanto, devido ao custo elevado, capacitação especializada de profissionais, e softwares desenvolvidos para cada finalidade, existe a necessidade da utilização de medidas que possam ser indicativas de risco relacionado ao excesso de gordura corporal, como as medidas antropométricas (4). A distribuição de gordura relaciona-se ao prognóstico de risco para a saúde, sendo a adiposidade central associada fortemente com diversos fatores de risco para doenças cardiovasculares (5).

Outro método prático é sugerido para a detecção de gordura corporal: a circunferência do pescoço (CP). É relativamente novo, simples, realizada na altura média do pescoço que não sofre interferência por movimentos respiratórios ou por distensão pós-prandial como a circunferência da cintura, e possui boa correlação com o acúmulo subcutâneo de gordura na região superior do corpo (6)

Baseado nestas evidências e na necessidade de mais estudos utilizando indicadores antropométricos de fácil aplicabilidade e baixo custo que possam ser aliados na prática clínica e em estudos epidemiológicos como marcadores de excesso de peso e do risco cardiovascular, o presente estudo tem como objetivo investigar a associação entre a CP e os fatores de risco cardiovascular em crianças e adolescentes atendidos no ambulatório de pediatria de um Hospital Universitário em Recife, PE, Brasil.

\section{Métodos}

Estudo transversal, desenvolvido com crianças e adolescentes acompanhados no ambulatório de nutrição de um hospital universitário na cidade de Recife, PE, Brasil. Os pacientes desse ambulatório são encaminhados via regulação ou através de encaminhamentos durante a internação no próprio hospital. O ambulatório funciona duas vezes por semana atendendo cerca de 15 pacientes por dia. Foram excluídas da pesquisa crianças com idade igual ou menor que cinco anos e maior ou igual a 18 anos, presença de comorbidades cujas caracteristicas pudessem interferir nos resultados antropométricos e demais variáveis como: paralisia cerebral; portadores de erros inatos do metabolismo; doença renal crônica; hepatopatia e fazer uso de corticoterapia; e não ter exames laboratoriais dos últimos três meses constando perfil lipidico: High Density Lipoprotein (HDL), Low Density Lipoprotein (LDL), Very Low Density Lipoprotein (VLDL) e triglicerideos.

Foram utilizadas como medidas antropométricas: peso, altura, CC e CP. O questionário foi aplicado e respondido com o responsável. Ele enquadrava questões sobre nivel de instrução e nível socioeconômico dos pais, histórico familiar de doença cardiovascular e/ou diabetes mellitus, prática de atividade fisica e tempo de tela. Para avaliação do 
tempo de tela, os indivíduos foram questionados quanto ao tempo dispendido em frente à TV, ao computador, ao videogame e ao celular/tablet e, também, em relação ao tempo, se $<2$ horas ou $>2$ horas por dia, segundo recomendação da Academia Americana de Pediatria (7). Quanto à atividade física, foram questionados quanto à prática ou não e quanto à duração em minutos.

O peso e a altura foram aferidos em dualidade, de acordo com os critérios de Lohman (8), a partir dessas medidas, determinou-se o escore Z do IMC para idade, considerando-se: baixo peso: < Escore - 2,0; eutrofia: $\geq$ Escore Z -2 e $\leq 1$; sobrepeso: > Escore Z +1 e $\leq+2$; Obesidade: > Escore Z +2 (9). Após essa classificação, a amostra foi separada em dois grupos: com excesso de peso, aqueles que ficaram classificados em sobrepeso e obesidade; e sem excesso de peso, aqueles que foram classificados em baixo peso ou eutrofia. A CC foi medida com fita métrica inelástica no ponto médio entre a crista iliaca e a última costela. A CP foi aferida na altura média do pescoço. Para classificação da CP foi utilizado o ponto de corte proposto por Lou et al. (10), ( $n=2.847)$ e utilizou o seguinte ponto de corte: meninos: 27,4 a 31,3 cm e meninas: 26,3 a 31,4 cm.

Na avaliação das variáveis bioquímicas, adotou-se os valores de referência recomendados pela Sociedade Brasileira de Cardiologia (11).

Para análise estatística foram utilizados os programas SPSS for Windows 13 e Epi-Info versão 3.5.4. As variáveis contínuas foram testadas quanto à normalidade da distribuição, pelo teste de Kolmogorov-Smirnov, e foram descritas na forma de mediana e intervalo interquartílico. $\mathrm{Na}$ descrição das proporções, a distribuição binomial foi aproximada à distribuição normal, pelo intervalo de confiança de 95\%. Para verificar se houve associação entre a CP e variáveis sociodemográficas e de estilo de vida (tempo de tela e prática de atividade física) utilizou-se o teste qui-quadrado de Pearson. Para avaliar a relação entre a CP, o perfil lipídico e variáveis antropométricas foi utilizado a correlação Spearman. Foi considerado o nível de significância de 5,0\% para rejeição de hipótese de nulidade.
A pesquisa teve aprovação do Comitê de Ética em Pesquisa envolvendo seres humanos da Universidade Federal de Pernambuco, com parecer consubstanciado $n .^{\circ} 2.576 .132$, e a coleta de dados foi iniciada após assinatura do Termo de Consentimento Livre e Esclarecido pelos pais ou responsáveis.

\section{Resultados}

A amostra foi por conveniência, constituida por 159 pacientes. Desses, 13 estavam com informações incompletas no prontuário e dois se recusaram a participar, apresentando um $n$ final de 144 pacientes. A idade média foi 11,0 $\pm 3,0$ anos, e desses, 90 (62,5\%) eram adolescentes e a maioria $(73=51,4 \%)$ do sexo masculino. Quanto à realização de atividade física, 109 (76\%) pacientes relataram praticar algum tipo de atividade física e a mediana da prática foi de 3 vezes por semana (IQ: 2-5) com mediana da duração de 60 minutos/ semana (IQ: 60-120). Avaliando o histórico familiar de doença cardiovascular observa-se que 115(79,9\%) pacientes relataram essa presença, e 102 (71,5\%) tinham algum parente direto com diabetes mellitus. 102 (71,3\%) relataram tempo de tela maior que duas horas e o equipamento eletrônico mais utilizado por 53 (36,8\%) dos entrevistados foi o celular/tablet. As características das crianças e dos adolescentes quanto às variáveis demográficas, socioeconômicas, clínicas e de estilo de vida estão descritas na Tabela 1.

Em relação ao estado nutricional, a frequência foi de pacientes sem excesso de peso (9o $=62.5 \%$ ) classificados de acordo com o IMC e 72 (50\%) deles apresentaram circunferência da cintura elevada. Já quanto à circunferência do pescoço, 53 (37,6\%) apresentaram a CP elevada. Quando estratificado de acordo com o sexo, 48 (33,8\%) meninas apresentaram CP elevada e 59 $(41,1 \%)$ meninos também tinham elevação dessa medida. Quanto às variáveis bioquimicas, a fração lipídica que teve maior alteração foi triglicerideo, 75 (52,4\%) pacientes apresentando valores elevados, seguido do Colesterol Total (CT), sendo 60 $(41,7 \%)$ pacientes com valores elevados. 
TABELA 1 - Caracteristicas socioeconômicas, demográficas e clínicas de crianças e adolescentes atendidos ambulatorialmente em um hospital universitário em Pernambuco, 2018.

\begin{tabular}{|c|c|c|}
\hline Variáveis & $\mathbf{n}$ & $\%$ \\
\hline Adolescentes & 90 & 62,5 \\
\hline Sexo Masculino & 74 & 51,4 \\
\hline \multicolumn{3}{|l|}{ Procedência } \\
\hline Recife & 35 & 24,2 \\
\hline Região Metropolitana & 46 & 31,9 \\
\hline Interior & 63 & 43.9 \\
\hline Escolaridade do Responsável $\geq 8$ anos & 98 & 68,1 \\
\hline Renda Familiar $\leq 1$ salário-mínimo & 94 & 65.3 \\
\hline $\begin{array}{l}\text { História familiar de doença } \\
\text { cardiovascular }\end{array}$ & 115 & 79.9 \\
\hline História familiar de diabetes mellitus & 103 & 71,5 \\
\hline Prática de atividade física & 76 & 52,8 \\
\hline Tempo de tela $\geq 2$ horas & 103 & 71,5 \\
\hline
\end{tabular}

Ao avaliar a associação da CP com variáveis sociodemográficas e estado nutricional foi possivel observar que o sexo masculino apresentou maiores valores dessa medida $(59=41,1 \%)$ e houve associação significativa com o estado nutricional, onde 22 (24,8\%) daqueles com excesso de peso apresentaram CP elevada. Em relação à prática de atividade física, 19 (37.3\%) dos que apresentaram CP elevada relataram não fazer nenhum tipo de atividade física, e 22 (42,6\%) relataram ter um tempo de tela maior que duas horas/dia (Tabela 2). Não foi visto associação estatisticamente significante da medida da CP com essas variáveis, no entanto, foi possivel observar que a frequência de alteração na CP aumenta com o aumento do tempo de tela.
TABELA 2 - Associação da CP com variáveis sociodemográficas e estilo de vida em crianças e adolescentes atendidos ambulatorialmente em um hospital universitário em Pernambuco, 2018.

\begin{tabular}{lccc}
\hline & $\begin{array}{c}\text { CP } \\
\text { Normal, } \\
\mathbf{n}(\%)\end{array}$ & $\begin{array}{c}\text { CP } \\
\text { Elevada, } \\
\mathbf{n}(\%)\end{array}$ & $\mathbf{p}$ \\
\hline Adolescentes & $53(60,2)$ & $35(39,8)$ & 0,610 \\
Sexo masculino & $43(58,9)$ & $30(41,1)$ & 0,473 \\
$\begin{array}{l}\text { Prática de } \\
\text { atividade física }\end{array}$ & $46(62,2)$ & $28(37,8)$ & 1,000 \\
$\begin{array}{l}\text { Tempo de } \\
\text { Tela } \geq 2 \text { horas }\end{array}$ & $58(57,4)$ & $43(42,6)$ & 0,080 \\
$\begin{array}{l}\text { Estado Nutricional: } \\
\text { com excesso de } \\
\text { peso }\end{array}$ & 19(13,4) & 35(24,8) & 0,001 \\
\hline $\begin{array}{l}\text { CP, circunferência do pescoço. 'Teste qui-quadrado } \\
\text { de Pearson. }\end{array}$ & &
\end{tabular}

$\mathrm{Na}$ análise da CP com perfil lipídico e outras variáveis antropométricas, nota-se que houve relação com HDL $(p=0,001), \operatorname{VLDL}(p=0,005)$ e triglicerideos $(p=0,02)$. Na correlação com IMC e $C C$, percebe-se que também houve associação $(p=0,001)$ (Tabela 3$)$

TABELA 3 - Correlação da circunferência do pescoço com perfil lipídico e variáveis antropométricas em crianças e adolescentes atendidos ambulatorialmente em um hospital universitário em Pernambuco, 2018.

\begin{tabular}{llc}
\hline & \multicolumn{2}{c}{$\begin{array}{c}\text { Circunferência do } \\
\text { Pescoço }\end{array}$} \\
\hline Rho & p \\
\hline CT, mg/dl & $-0,073$ & 0,391 \\
LDL, mg/dl & $-0,046$ & 0,589 \\
HDL, mg/dl & $-0,268$ & 0,001 \\
VLDL, mg/dl & 0,245 & 0,005 \\
Triglicerideo, mg/dl & 0,184 & 0,029 \\
IMC, kg/m² & 0,745 & $<0,001$ \\
CC, $\mathrm{cm}$ & 0,811 & $<0,001$ \\
\hline
\end{tabular}

CC, Circunferência da Cintura; CT, colesterol total; IMC, indice de massa corporal; HDL, lipoproteina de alta densidade; LDL, lipoproteína de baixa densidade; VLDL, lipoproteinas de muito baixa densidade. Correlação de Spearman. 


\section{Discussão}

O presente estudo teve o intuito de verificar se a CP está associada a fatores de risco cardiovascular, através de pontos de cortes citados na literatura. Constata-se em nossa amostra que um elevado percentual de pacientes apresentou a CP elevada (37,6\%), sendo a CP associada com a gordura corporal. Esse fato talvez seja explicado pelo processo de transição nutricional que vem ocorrendo no Brasil, mostrando que mesmo com a maioria da população do estudo ainda eutrófica, percebe-se a ocorrência de mudanças no estado nutricional decorrentes do estilo de vida que parece estar se modificando.

Quanto ao tempo de tela, 71,5\% relataram passar mais de duas horas por dia em frente a algum aparelho eletrônico. Confrontando com outros resultados disponiveis na literatura, são encontradas proporções mais baixas (12), e mais altas (13, 14), de adolescentes expostos ao comportamento sedentário. Podendo essa diferença ser atribuída às formas com que esse comportamento foi medido e classificado, já que existe uma variação nos pontos de corte que vão de $\geq 2$ horas a $\geq 6$ horas/dia. Também é válido ressaltar que, nos estudos que consideram a televisão como indicador do comportamento sedentário, a prevalência varia de $27 \%$ a $65 \%(15,16)$, valores maiores que o encontrado no presente estudo, que foi de $20,4 \%$. Entretanto, poucos estudos avaliaram a frequência de exposição ao tempo em tablets e smartphones, mesmo apesar do padrão de utilização de aparelhos eletrônicos ter mudado nos últimos anos. Na presente população, foi visto um valor alto de uso desses aparelhos (52 = $36,8 \%$ ). Percebe-se assim, como é preocupante a alta frequência de jovens expostos ao CS.

Em estudo realizado por Lourenço et al., foi encontrado uma prevalência de comportamento sedentário de $81,6 \%$, sendo maior no sexo masculino (17). Em outro estudo, que verificou associação entre tempo de tela e padrões alimentares, foi visto uma prevalência de alto tempo de tela $(39,1 \%)$, sendo o padrão alimentar encontrado o obesogênico (18).

Segundo Lucena e Cols, prevalências elevadas de tempo excessivo de tela, frequentemente observadas em adolescentes, podem ser decorrentes das mudanças ocorridas na sociedade nas últimas duas a três décadas, como, por exemplo, o crescimento econômico que permitiu às famílias, sobretudo as de renda média-baixa, mais acesso à televisão, computador, mais uso da internet no tempo de lazer (p. ex., interagir nas redes sociais) e redução dos espaços públicos para a prática de atividades físicas, associada à insegurança observada nos grandes centros urbanos (19).

Foi encontrado correlação negativa entre HDLe $C P(r=-0,268 p=0,001)$, corroborando com o estudo de Barreto, Gurgel e Souza (2016) (20), que encontraram que para cada aumento de um centímetro de CP ocorre a diminuição da concentração de 1,05mg/dL HDL-c. Nielsen et al. (21), demonstraram que a parte superior do tecido adiposo subcutâneo é responsável pela maior liberação de ácidos graxos livres do que a gordura visceral particularmente em individuos obesos, o que pode explicar essa correlação. Essa gordura subcutânea da parte superior do corpo é facilmente avaliada pela $\mathrm{CP}$, podendo ser um excelente preditor de risco cardiovascular e de obesidade (22).

Se analisarmos o histórico familiar de doenças cardiovasculares na população do presente estudo, é possivel perceber que esse indice é bastante elevado (79,9\%). Bao et al. (23), foram os primeiros a avaliar a associação de doença arterial coronariana e o perfil de risco cardiovascular em uma coorte de descendentes, que foram acompanhados da infância até a vida adulta. Nesse estudo, os filhos de individuos com doença coronariana apresentaram peso, níveis de CT, LDL-C, glicose e insulina superiores aos da descendência de pais sem doença arterial coronariana manifesta. Alguns estudiosos consideram que a ocorrência de outros fatores mais conhecidos como hipertensão arterial sistêmica (HAS), diabetes mellitus (DM), obesidade e outras doenças metabólicas também deve ser importante na hora de obter o histórico familiar para aterosclerose (24).

Não houve diferença estatistica quando correlacionado a CP com CT e com LDL, assim como no estudo de Pereira et al. (25), que compararam essas variáveis em grupos de adolescentes do 
sexo feminino, divididos em obesos e não obesos. A explicação pode vir do fato de que, no grupo com obesidade abdominal, os níveis de HDL-c serem mais baixos e os de LDL-c mais altos, não alterando, assim, o valor de colesterol total. Ressalta-se o fato que tal achado não descarta a importância do monitoramento precoce do CT.

Kurtoglu et al. (26), ao avaliarem a correlação da CP com marcadores da síndrome metabólica de acordo com sexo e estádio puberal, encontraram que no sexo masculino a CP correlacionou-se positivamente com pressão arterial sistólica e diastólica, glicemia, CT e triglicerídeo nos pré-púberes. No presente estudo também houve correlação positiva significativa entre CP e triglicerídeo $(r=$ $0,184 \mathrm{p}=0,029$ ), em ambos os sexos, porém não foi avaliado de acordo com estádio puberal.

No que concerne à medida da CP, existem poucos estudos associando-a com estado nutricional, mas os poucos existentes sugerem que essa é uma boa medida para determinar sobrepeso e obesidade em crianças e adolescentes. Em estudo realizado por Nafiu et al. (27), foi observado que a CP tinha melhor correlação com IMC e CC em meninos do que em meninas. Já no presente estudo, observou-se que, apesar de moderada, houve correlação entre CP e IMC $(r=0,745 p=<0,001)$, e forte correlação com CC $(\mathrm{r}=0,811 \mathrm{p}=<0,001)$ e CP, porém não foi avaliada a diferença entre os sexos.

A CP é um índice confiável para determinar a obesidade, devido às elevadas correlações encontradas entre CP e IMC. Ao mesmo tempo, estudos viram que a correlação entre CP e percentual de gordura corporal é baixa, significando que essa é uma medida de acúmulo de gordura desproporcional, em vez de uma medida geral de obesidade (28). Sabe-se que o IMC é um bom parâmetro para diagnosticar obesidade, porém ele não é um bom indicador de distribuição de gordura corporal, sendo já bem estabelecida a relação entre gordura abdominal e maiores riscos cardiovasculares e distúrbios metabólicos como resistência à insulina e intolerância à glicose (29): Esse fato reforça a importância de não serem utilizadas medidas antropométricas isoladas para diagnóstico nutricional.
Os achados do presente estudo indicaram que há associação significativa entre CP e medidas antropométricas classificadas como elevadas, corroborando com a literatura, que traz que tal medida pode ser uma ferramenta útil para identificar sobrepeso ou obesidade e importante preditor de problemas de saúde cardiovascular, já que se correlaciona também com perfil lipídico. Ressalta- se a necessidade de mais estudos a fim de determinar o ponto de corte da CP na população brasileira pediátrica para facilitar e padronizar o seu uso.

\section{Notas}

\section{Apoio financeiro}

Este estudo não recebeu apoio financeiro de fontes externas.

\section{Declaração de conflito de interesses}

Os autores declaram não haver conflitos de interesses relevantes ao conteúdo deste estudo.

\section{Contribuições dos autores}

Todos os autores fizeram contribuições substanciais para concepção, ou delineamento, ou aquisição, ou análise ou interpretação de dados; e redação do trabalho ou revisão crítica; e aprovação final da versão para publicação.

\section{Disponibilidade dos dados e responsabilidade pelos resultados}

Todos os autores declaram ter tido total acesso aos dados obtidos e assumem completa responsabilidade pela integridade destes resultados.

\section{Referências}

1. Bloch KV, Klein CH, Szklo M, Kuschnir MCC, Abreu G de A, Barufaldi LA, et al. Erica: Prevalência de hipertensão arterial e obesidade em adolescentes brasileiros. Rev. Saude Publica. 2016;50(suppl 1):9s. https://doi. org/10.1590/S01518-8787.2016050006685

2. Pinho L., Santos SHS, Caldeira AP, Botelho ACC, Flávio EF. Excesso de peso e consumo alimentar em adolescentes de escolas públicas no norte de Minas Gerais. Cien Saude Colet. 2014;(19):67-74. https://orcid. org/0000-0002-2947-5806

3. Ribas SA, Silva LCS. Fatores de risco cardiovascular e fatores associados em escolares do Município de Belém, Pará, Brasil. Cad. Saúde Pública. 2014:30(3):57786.https://doi.org/10.1590/0102-311X00129812 
4. Pelegrini A, Silva DAS, Silva JMF, Grigollo L, Petroski EL. Indicadores antropométricos de obesidade na predição de gordura corporal elevada em adolescentes. Rev. Paulista de Pediatr. 2015:33(1):56-62. https://doi. org/10.1016/j.rpped.2014.06.007

5. Magalhães EI S, Sant'ana LF R, Priore SE, Franceschini S CC. Perimetro da cintura, Relação cintura/estatura e perimetro do pescoço como parâmetros na avaliação da obesidade centralem crianças. Rev. Paul. Pediatr. 2014:32(3)27382. https://doi.org/10.1590/0103-0582201432320

6. Preis SR, Massaro JM, Hoffmann U, D'Agostino RB, Levy D, Robins SJ, et al. Neck circumference as a novel measure of cardiometabolic risk: The framingham heart study. J. Clin. Endocrinol. Metab. 2010:95(8):3701-10.

7. American Academy of Pediatrics. Committee on Public Education. American Academy of Pediatrics: Children, adolescents, and television. Pediatrics. 2001;107(2):4236. https://doi.org/10.1542/peds.107.2.423

8. Lohman TG, Roche AF, Martorell R. Anthrometric standardization reference manual. Champaign: Human Kinetics; 1988.

9. Onis M de, Onyango AW, Borghi E, Amani S, Chizuru N, Siekmann J. Development of a WHO growth reference for school-aged children and adolescents. Bull. World Health Organ. [Online]. 2007 Sep 1 lacesso em 21 jan. 2020];85(09):660-7. Disponivel em: http://www.who. int/bulletin/volumes/85/9/07-043497.pdf

10. Lou DH, Yin FZ, Wang R, Ma CM, Liu XL, Lu Q. Neck circumference is an accurate and simple index for evaluating overweight and obesity in Han children. Ann. Hum. Biol. 2012;39(2):161-5. https://doi.org/10.31 $\underline{09 / 03014460.2012 .660990}$

11. Faludi AA, Izar MCO, Saraiva JFK, Chacra APM, Bianco HT, Afiune Neto A et al. Atualização da Diretriz Brasileira de Dislipidemias e Prevenção da Aterosclerose - 2017. Arq Bras Cardiol 2017; 109(2Supl.1):1-76. https://doi. org/10.5935/abc.20170121

12. Dias PJP, Domingos IP, Ferreira MG, Muraro AP, Sichieri R, Silva RMV. Prevalência e fatores associados aos comportamentos sedentários em adolescentes. Rev. Saude Publica. 2014;48(2):266-74. https://doi. org/10.1590/So034-8910.2014048004635

13. Santos A, Andaki ACR, Santos Amorim PR Dos, Mendes EL. Fatores associados ao comportamento sedentário em escolares de 9-12 anos de idade. Motriz. Rev. Educ. Fis. 2013;19(3 SUPPL):25-34. http://dx.doi. org/10.1590/S1980-65742013000700005

14. Coelho LG, Cândido APC, Machado-Coelho GLL, Freitas SN de. Association between nutritional status, food habits and physical activity level in schoolchildren. J. Pediatr. (Rio. J). 2012;88(5):406-12. http://dx.doi. org/10.2223/JPED.2211

15. Camelo LV, Rodrigues JFC; Giatti L, Barreto SM. Lazer sedentário e consumo de alimentos entre adolescentes brasileiros: Pesquisa Nacional de Saúde do Escolar (PeNSE), 2009. Cad. Saúde Pública. 2012;28(11):2155-62. https://doi.org/10.1590/S0102-311X2012001100015
16. Ferreira WF, Rombaldi AJ, Ricardo LIC, Hallal PC, Azevedo MR. Prevalência de comportamento sedentário de escolares e fatores associados. Rev. Paul. Pediatr. 2016:34(1):56-63. http://dx.doi.org/10.1016/j. rppede.2015.09.002

17. Lourenço CLM, Zanetti HR, Amorim PRS, Mota JAPS, Mendes EL. Comportamento sedentário em adolescentes: prevalência e fatores associados. R. bras. Ci. e Mov 2018;26(3):23-32.

18. Pinho MGM, Adami F, Benedet J, Vasconcelos FAG. Associação entre tempo de tela e padrões alimentares com sobrepeso/obesidade em adolescentes. Rev de Nutr. 2017:30(3)377-89. https://doi.org/10.1590/167898652017000300010

19. Lucena JMS, Cheng LA, Cavalcante TLM, Silva VA, Junior JCF. Prevalência do tempo excessivo de tela e fatores associados em adolescentes. Rev. Paul. de Pediatr. 2015:33(4):407-14. http://dx.doi.org/10.1016/j. rpped.2015.04.001

20. Souza MFC, Gurgel RQ, Barreto IDC, Shanmugam S. Neck circumference as screening measure for identifying adolescents with overweight and obesity. J. Hum. Growth Dev. 2016;26(2):260-266. https://doi. org/10.7322/jhgd.119302

21. Nielsen S, Hensrud DD, Jensen MD, Nielsen S, Guo Z, Johnson CM, et al. Splanchnic lipolysis in human obesity Find the latest version: Splanchnic lipolysis in human obesity. J. Clin. Invest. 2004:113(11)1582-8. https://doi.org/10.1172/jci21047

22. Souza MFC. Identificação de pontos de corte da circunferência do pescoço para determinação dos niveis de excesso de peso e predição do risco cardiometabólico em adolescentes [tese]. Sergipe. Universidade Federal de Sergipe, 2016. 67p.

23. Bao W, Srinivasan SR, Valdez R, Greenlund KJ, Wattigney WA, Berenson GS. Longitudinal changes in cardiovascular risk from childhood to young adulthood in offspring of parents with coronary artery disease: the Bogalusa Heart Study. JAMA. 1997:278(21):1749-54. doi:10.1001/jama.1997.03550210047037

24. Moura IH, Vieira EES, Silva GRF, Carvalho RBN, Silva ARV. Prevalência de hipertensão arterial e seus fatores de risco em adolescentes. Acta Paul Enferm. 2015;28(1):81-6. http://dx.doi.org/10.1590/1982$\underline{0194201500014}$

25. Pereira PF, Serrano HMS, Carvalho GQ, Lamounier JA, Peluzio M do CG, Franceschini S do CC, et al. Circunferência da cintura e relação cintura/estatura: Úteis para identifcar risco metabólico em adolescentes do sexo feminino? Rev. Paul. Pediatr. 2011;29(3):372-7. https://doi.org/10.1590/S0103-05822011000300011

26. Hatipoglu N, Mazicioglu MM, Kurtoglu S, Kendirci M. Neck circumference: An additional tool of screening overweight and obesity in childhood. Eur. J. Pediatr. 2010;169(6):733-9. https://doi.org/10.1007/s00431$009-1104-z$ 
27. Nafiu OO, Burke C, Lee J, Voepel-Lewis T, Malviya S, Tremper KK. Neck circunference as a screening measure for identifying childrn with high body mass index. Pediatrics. 2010;126(2):e306-10. https://doi.org/10.1542/ peds.2010-0242

28. Mazicioglu MM, Kurtoglu S, Ozturk A, Hatipoglu N, Cicek B, Ustunbas HB. Percentiles and mean values for neck circumference in Turkish children aged 6-18 years. Acta Pediatr. 2010;99:1847-53.

29. Hatipoglu N, et al. Relationship between neck circunference and non-alcoholic fatty liver disease in childhood obesity. Journ of Clini Res in Pediat Endocrin. 2016:8(1):32-9.

\section{Ávilla Monalisa Silva de Oliveira}

Especialista em Nutrição Clínica pelo Hospital das Clinicas da Universidade Federal de Pernambuco (HC-UFPE), em Recife, PE, Brasil.

\section{Skalyt Lee Barbosa e Silva}

Especialista em Nutrição Clínica pelo Hospital das Clínicas da Universidade Federal de Pernambuco (HC-UFPE), em Recife, PE, Brasil.

\section{Lílian Caroline de Souza e Silva}

Especialista em Nutrição Clínica pelo Hospital das Clínicas da Universidade Federal de Pernambuco (HC-UFPE), em Recife, PE, Brasil.

\section{Ilma Kruze Grande de Arruda}

Doutora em Nutrição pela Universidade Federal de Pernambuco (UFPE), em Recife, PE, Brasil; professora titular da Universidade Federal de Pernambuco (UFPE), em Recife, PE, Brasil.

\section{Endereço para correspondência}

\section{Ávilla Monalisa Silva de Oliveira}

Universidade Federal de Pernambuco

Av. Prof. Moraes Rego, 1235

Cidade Universitária, 50670-901

Recife, PE, Brasil 